hep-th/9702038, IASSNS-HEP-97/7

\title{
RG Fixed Points in Six Dimensions via Branes at Orbifold Singularities
}

\author{
Kenneth Intriligator* \\ School of Natural Sciences \\ Institute for Advanced Study \\ Princeton, NJ 08540, USA
}

\begin{abstract}
We discuss a set of generalized, necessary conditions for non-trivial, interacting fixed points in six dimensional supersymmetric field theories. We use string theory to argue for the existence of infinite families of interacting RG fixed point theories. The theories are based on certain gauge groups and matter content, which we identify, along with additional tensor multiplets. They are conjectured to arise in the world-volume of Type I D5 branes at orbifold singularities.
\end{abstract}

$2 / 97$

* On leave 1996-1997 from Department of Physics, University of California, San Diego. 


\section{Introduction}

D branes have quantum field theories living in their world volume; see [1] for a recent review with references. For example, in the type I theory, a $S O(32)$ instanton of zero size, which is a D5 brane, has an $S p(1) \cong S U(2)$ gauge theory with $N_{f}=16$ fundamentals living in its world volume [2]. This $6 \mathrm{~d}$ gauge theory is free at long distances and, in the ultraviolet, more data, such as string theory, is needed to obtain a sensible theoryl the $E_{8}$ small instanton also leads to a six dimensional world-volume theory in which gravity decouples at long distance. This case appears more exotic - like a "tensionless string" [3-6] - though there is evidence that it is an interacting local quantum field theory at a non-trivial RG fixed point [4.6-10]. The existence of other non-trivial fixed points of the renormalization group in six dimensions was discussed in [11]. For example, it was there argued via string theory that $S U(2)$ with $N_{f}=4$ and $N_{f}=10$ fundamental flavors, along with a tensor multiplet to cancel gauge anomalies, have non-trivial fixed points. It was further conjectured that such fixed points actually exist for all $N_{f}<16$ [11]. It remains a challenge to formulate and eventually understand non-trivial six dimensional fixed points directly in terms of quantum field theory, without having to appeal to stringy considerations.

Here we will construct new, six-dimensional fixed point theories by considering type I D5 branes when the Dirichlet location $\vec{x} \in R^{4}$, namely the location of the small $S O(32)$ instantons, is on top of an orbifold singularity. We present evidence that, much as with the small $E_{8}$ instanton 四, there is a "tensionless string" type transition region where a "Higgs branch," associated with the instanton moduli, joins to a "Coulomb branch," associated with massless tensor multiplets whose scalar components can get expectation values. The theories on the Coulomb branch are $6 \mathrm{~d}$ gauge theories, with gauge groups and matter content which we identify, along with the extra tensor multiplets. We argue that these gauge theories with extra tensor multiplets have non-trivial RG fixed points at the origin of their moduli space.

The simplest example of the "transition" from the Higgs branch to the Coulomb branch occurs for four D5 branes at a $Z_{2}$ orbifold singularity. With fewer than four instantons, there is no Coulomb branch. On the Higgs branch, the theory appears to be "exotic." Along the Coulomb branch, on the other hand, the theory is simply given by an $S p(4)$ gauge theory with 16 fundamentals and a tensor multiplet. We can approach the

1 I would like to thank N. Seiberg and E. Witten for instructive comments on this point. 
RG fixed point at the origin, where the Higgs and Coulomb branches touch, from along the Coulomb branch. Precisely this "transition" from the Higgs to the Coulomb branch also appeared recently in [12], where the transition was discussed in detail in terms of $\mathrm{F}$ theory.

In the next section, we will discuss a generalized version of the anomaly condition of [11] for non-trivial RG fixed points in six dimensions. In sect. 3, we review aspects of small $S O(N)$ instantons. In sect. 4, we discuss small $S O(N)$ instantons on a $Z_{2}$ orbifold singularity. Following the discussion in [13], there are two situations: with and without "vector structure2." We first discuss the case without vector structure, which is simpler; the essential features in this case already appeared in [14,13]. In sect. 4.2, we consider the case with vector structure and present evidence that there is a Coulomb branch associated with the gauge group $S p(K) \times S p(K+n-4)$ with $16-2 n$ matter fields in the $(\square, \mathbf{1}), 2 n$ matter fields in the $(\mathbf{1}, \square)$, one matter field in the $(\square, \square)$, and a tensor multiplet. Here $n$ is an arbitrary integer between 0 and 8 and $K$ is an arbitrary integer with $K+n \geq 4$. One of the gauge groups, say $S p(K+n-4)$, is IR free, while the other flows to an interacting fixed point. At the fixed point, the theory is $S p(K)$ with matter $(2 K+8) \cdot \square$ and a tensor multiplet. String theory proves that these fixed points really exist for all $K$. In sect. 5 , we discuss the situation for small $S O(N)$ instantons on a $Z_{M}$ orbifold singularity, with vector structure, for arbitrary $M$. We thus argue for the existence of two infinite families of nontrivial six dimensional fixed points involving gauge theories with extra tensor multiplets. In sect. 6 we discuss the case without vector structure. The gauge groups and matter hypermultiplets of all of these theories are given by the type I "quiver diagrams" of [15] with specific choices of quiver data.

\section{Six Dimensional Gauge Theories and Anomalies}

We will be interested in six dimensional theories with the minimal amount of supersymmetry. As discussed in [1], there can be interacting renormalization group fixed

2 This terminology is actually being misused here to refer, as discussed later, to the behavior of a flat connection at infinity. However, it was shown in [12] that there can be a non-trivial, "hidden obstruction" $\tilde{w}_{2}$ to vector structure even when the flat connection at infinity is trivial. In fact, on the Coulomb branch, the question of vector structure is ill-defined: the obstruction $\tilde{w}_{2}$ can change from being zero to non-zero [12]. For lack of better terminology, we will here continue to use "vector structure" in a sloppy sense. I am grateful to P.S. Aspinwall for a helpful correspondence on this issue. 
points which are related to gauge theories; a necessary condition for such a fixed point is that the quartic gauge anomaly can be cancelled. Here we will discuss the straightforward generalization of the conditions in [11] to non-simple gauge groups with more than one tensor multiplet.

The six dimensional gauge anomaly for a product gauge group $G=\prod_{a} G_{a}$ is

$$
\mathcal{A}=\sum_{a}\left(\operatorname{Tr} F_{a}^{4}-\sum_{i} n_{i} \operatorname{tr}_{i} F_{a}^{4}\right)-6 \sum_{a<b} \sum_{i<j} n_{i j} \operatorname{tr}_{i} F_{a}^{2} \operatorname{tr}_{j} F_{b}^{2} .
$$

Here $\operatorname{Tr}$ is in the adjoint representation, $\operatorname{tr}_{i}$ is in representation $R_{i}$ of $G_{a}, n_{i}$ is the number of matter fields in representation $R_{i}$, and $n_{i j}$ is the number of matter fields in representation $\left(R_{i}, R_{j}\right)$ of $G_{a} \times G_{b}$. This is as in [16], but with the gravitational parts dropped and a rescaling. The anomaly (2.1) will be of the form

$$
\mathcal{A}=\sum_{a} \alpha_{a} \operatorname{tr} F_{a}^{4}+\sum_{a b} c_{a b}\left(\operatorname{tr} F_{a}^{2}\right)\left(\operatorname{tr} F_{b}^{2}\right)
$$

with $\operatorname{tr}$ in the fundamental representation. Formulae which will be of later use in this regard are:

$$
\begin{array}{lcc}
S U(n): & \operatorname{Tr} F^{4}=2 n \operatorname{tr} F^{4}+6\left(\operatorname{tr} F_{2}\right)^{2}, & \operatorname{tr} \square^{4}=(n-8) \operatorname{tr} F^{4}+3\left(\operatorname{tr} F^{2}\right)^{2} ; \\
S p(n): & \operatorname{Tr} F^{4}=(2 n+8) \operatorname{tr} F^{4}+3\left(\operatorname{tr} F^{2}\right)^{2}, & \operatorname{tr} F^{4}=(2 n-8) \operatorname{tr} F^{4}+3\left(\operatorname{tr} F^{2}\right)^{2} .
\end{array}
$$

If $\mathcal{A}=0$, the theory is anomaly free and exists with a coupling constant parameter $g$. If $\alpha_{a}=0$ but $c_{a b} \neq 0$ in (2.2), there is an anomaly which can perhaps be cancelled by including tensor multiplets. Much as in [17], though without including gravity, the condition for being able to cancel the anomaly in a theory with $P$ tensor multiplets is

$$
\mathcal{A}=\sum_{i=1}^{P}\left(\sum_{a} C_{i a} \operatorname{tr} F_{a}^{2}\right)^{2}
$$

for some real constants $C_{i a}$. In particular, for $G$ simple and $P=1$ the condition on (2.2) is $\alpha=0$ and $c>0$ [11]. The anomaly cancellation occurs by coupling the scalar fields $\Phi_{i}$ in the tensor multiplets to the gauge fields by the interactions

$$
\sum_{i=1}^{P} \sum_{a} C_{i a} \Phi_{i} \operatorname{tr} F_{a}^{2} ;
$$

this leads to effective gauge couplings $g_{a}^{-2}(\Phi)=g_{a, c l}^{-2}+\sum_{i} C_{i a} \Phi_{i}$.

When $\alpha_{a}=0$ but (2.4) is not satisfied, the anomalies can possibly be cancelled by including gravity, as in [16]. If any $\alpha_{a} \neq 0$, the theory is inconsistent and can not be cured by adding any more fields - even gravity. 


\section{Small Instantons and the hyper-Kahler quotient}

As discussed in [2], there is an interesting mathematical fact with a beautiful connection to physics. The mathematical fact is that the moduli space of $K S O(N)$ instantons, which is a hyper-Kahler space, has a "hyper-Kahler quotient" construction as the Higgs branch of a gauge theory with 8 super-charges (i.e. $N=1$ supersymmetry in $6 \mathrm{~d}$ or $5 \mathrm{~d}$, $N=2$ in $4 \mathrm{~d}, N=4$ in $3 \mathrm{~d}$, etc. $)^{3}$. The gauge group is $S p(K)$ and the matter content consists of $N$ half-hypermultiplets in the $\square$ and a hypermultiplet in the $\square$.

The beautiful connection to physics is that this gauge theory, with $N=32$, is physically realized: it lives on the world volume of $K$ type I D5 branes at the same point $\mathbf{x} \in R^{4}$ fixed by the Dirichlet boundary conditions. The anomaly $\mathcal{A}$ vanishes for any choice of $K$ [16]. The six dimensional $S p(K)$ theory exists as a low energy theory, with coupling constant $g$, which is IR free. In the UV more data, such as string theory, is needed to obtain a sensible theory.

\section{D5 branes at a $Z_{2}$ orbifold singularity}

Consider $S O(N)$ instantons on a $Z_{2}$ orbifold singularity. The physical data is specified by an integer $K$, related to the number of instantons, as well as the possibility of having a non-trivial flat connection at infinity. Corresponding to the flat connection at infinity is an $S O(N)$ group element $\rho_{\infty}$ which has to satisfy $\rho_{\infty}^{2}=1$; the possibility of non-trivial $\rho_{\infty}$ is allowed by the $Z_{2}$ orbifold identification. Actually, as discussed in [13], there is another possibility in string theory which makes use of the fact that the gauge group is really $\operatorname{Spin}(32) / Z_{2}$ : we could have $\rho_{\infty}^{2}=-1$. As in [13], we will refer to the situation with $\rho_{\infty}^{2}=1$ as the case with vector structure and the situation with $\rho_{\infty}^{2}=-1$ as the case without vector structure. We will first discuss the case without vector structure, as it is simpler, with the main points already appearing in [14,13].

3 This can be generalized to the moduli space of $U(N)$ and $S p(N)$ instantons, with the moduli spaces described as the Higgs branches of $U(K)$ and $S O(K)$ gauge theories, respectively. The generalization to the exceptional gauge groups, and in particular $E_{8}$, appears problematic; this is connected with the fact that the physics of the small $E_{8}$ instanton is exotic. 


\subsection{The case without vector structure}

Consider generally $\operatorname{Spin}(N) / Z_{2}$, for even $N$, when $\rho_{\infty}$ has $\frac{1}{2} N$ eigenvalues $i$ and $-i$. The second Chern class of the gauge connection, i.e. the instanton number, is given by

$$
I=K+\frac{N}{32}
$$

where $K$ is an integer and the last term is a contribution coming from the non-trivial connection at infinity, as explained in [13]. The moduli space of instantons with this $\rho_{\infty}$, which is a hyper-Kahler space, has dimension in hypermultiplet (i.e. quaternionic) units (one quarter the real dimension) given by an index theorem to be

$$
\operatorname{dim}\left(\mathcal{M}_{\text {Inst }}\right)=(N-2) I-\frac{N(N-2)}{32}=(N-2) K,
$$

where $-N(N-2) / 32$ is the contribution from the $\eta$ invariant, which was discussed for the physical case $N=32$ in [13].

The moduli space $\mathcal{M}_{\text {Inst }}$ is conjectured to be isomorphic to the Higgs branch of a $U(2 K)$ gauge theory with $\frac{1}{2} N$ matter fields in the $\square$ and two in the $\boxminus$. These gauge theories were first derived in [14] via an orientifold construction of type I on K3. Note that $\operatorname{dim}\left(\mathcal{M}_{H}\right)=\operatorname{dim}\left(\mathcal{M}_{\text {Inst }}\right)$, given by (4.2). Further, when a Fayet-Iliopoulos term $\vec{\zeta}$ (which is a real triplet under the $S U(2)_{R}$ associated with the Hyper-Kahler structure) is added for the overall $U(1)$ factor in $U(2 K), \mathcal{M}_{H} \cong \mathcal{M}_{\text {Inst }}$ for instantons on the orbifold with blowing up parameter $\vec{\zeta}$, much as in the $U(N)$ instanton case of [18, 15. 1 .

As a check on the conjecture that $\mathcal{M}_{H} \cong \mathcal{M}_{\text {Inst }}$, note that it is easy to identify the direction in the Higgs branch which corresponds to moving the $K$ point-like instantons away from the orbifold singularity: give the matter fields in the $\boxminus$ expectation values $E_{f} J$, where $E_{f}$ is a hypermultiplet, with $f=1,2$ a flavor index, and $J \equiv 1_{K} \otimes\left(i \sigma_{2}\right)$. This is a $D$-flat direction provided the $E_{f}$ satisfy the $D$ term equations associated with the overall $U(1)$, including the Fayet-Iliopoulos term. This problem with the $E_{f}$ is simply that of $U(1)$ with two electrons, which is precisely the theory whose Higgs branch is isomorphic to the $Z_{2}$ orbifold with blowing up parameter $\vec{\zeta}$ (i.e. the $A_{1}$ ALE or Eguchi-Hanson space)

4 As discussed in some detail in [15, 13], in 6d $U(1)$ factors with charged matter are anomalous and become massive via coupling to a scalar. The scalar is part of a hypermultiplet whose expectation value gives the Fayet-Iliopoulos term. In short, the $U(1)$ factors go away and the FayetIliopoulos parameter $\vec{\zeta}$ is replaced with the expectation value of an additional hypermultiplet. 
[19]. The Higgs branch associated with the $\left\langle E_{f}\right\rangle$ is thus the modulus for moving the $K$ point-like instantons around on the blown up orbifold. The point at the origin, $E_{1,2}=0$, corresponds to the point-like instantons sitting on the orbifold singularity. This point is only on the moduli space when the Fayet-Iliopoulos blowing up parameter $\vec{\zeta}$ is set to zero. At this point, the full $U(2 K)$ is unbroken. Away from this point, $U(2 K)$ is Higgsed to $S p(K)$ with the correct matter content, reviewed in the previous section, for describing the $K$ point-like instantons away from the orbifold singularity, or when the singularity is blown up with $\vec{\zeta} \neq 0$.

The anomaly (2.1) for the $U(2 K)$ theory, using (2.3), is $\mathcal{A}=\left(16-\frac{1}{2} N\right) \operatorname{tr} F^{4}$, and thus vanishes identically for the physical case of $N=32$. The theory is free at long distances.

\subsection{The case with vector structure.}

The element $\rho_{\infty} \in S O(N)$ is specified by the number $w_{0}$ of eigenvalues equal to +1 and the number $w_{1}$ eigenvalues equal to -1 , with $w_{0}+w_{1}=N$ and $w_{1}$ even in order to have $\rho_{\infty} \in S O(N)$. To have $\rho_{\infty}^{2}=1$ in $\operatorname{Spin}(N), w_{1}$ actually has to be a multiple of four. With this physical data, the second Chern class of the gauge connection, i.e. the instanton number, is given by

$$
I=K+\frac{w_{1}}{8}
$$

where $K$ is an integer and the last term is a contribution coming from the non-trivial connection at infinity, as explained in [13]. Note that, $I$ can be half-integral. The moduli space of such instantons has dimension in hypermultiplet units given by

$$
\operatorname{dim}\left(\mathcal{M}_{\text {Inst }}\right)=(N-2) I-\frac{1}{8} w_{0} w_{1}
$$

the last term is the $\eta$ invariant contribution.

The relevant gauge theory is $S p\left(v_{0}\right) \times S p\left(v_{1}\right)$ with $w_{0}$ half-hypermultiplets in the $(\square, \mathbf{1})$ representation, $w_{1}$ half-hypermultiplets in the $(\mathbf{1}, \square)$, and one in the $(\square, \square)$. This corresponds to one of the type I quiver diagrams of [15]. Using (2.3), the anomaly (2.1) is

$$
\mathcal{A}=\left(2 v_{0}+8-\frac{1}{2} w_{0}-2 v_{1}\right) \operatorname{tr} F_{0}^{4}+\left(2 v_{1}+8-\frac{1}{2} w_{1}-2 v_{0}\right) \operatorname{tr} F_{1}^{4}+3\left(\operatorname{tr} F_{0}^{2}-\operatorname{tr} F_{1}^{2}\right)^{2} .
$$

In general, this theory has a Higgs branch moduli space of vacua, $\mathcal{M}_{H}$, which is a hyperKahler space, where the hypermultiplets get expectation values. When there is enough 
matter to completely Higgs the gauge group, which will be the case in what follows, the dimension of the Higgs branch is the number of hyper minus vector multiplets:

$$
\operatorname{dim}\left(\mathcal{M}_{H}\right)=\sum_{\mu=0}^{1} w_{\mu} v_{\mu}+4 v_{0} v_{1}-\sum_{\mu=0}^{1} v_{\mu}\left(2 v_{\mu}+1\right)
$$

We conjecture that $\mathcal{M}_{\text {Inst }} \cong \mathcal{M}_{H}$ with the following relation between the $v_{\mu}$ and the physical data:

$$
\left.v_{0}=K, \quad v_{1}=K+\frac{w_{1}}{4} \quad \text { (Hyper }- \text { Kahler quotient }\right)
$$

Note that, with this identification, the instanton number (4.3) is given by

$$
I=\frac{1}{2}\left(v_{0}+v_{1}\right)
$$

which is quite natural upon considering the $S p\left(v_{\mu}\right)$ on the covering space of the $Z_{2}$ orbifold. Also, substituting in (4.7), it is seen that the dimensions in (4.4) and (4.6) agree.

As a further check on $\mathcal{M}_{H} \cong \mathcal{M}_{\text {Inst }}$, we note that the gauge theory properly has moduli which correspond to moving small instantons away from the $Z_{2}$ orbifold singularity: The $S p(K) \times S p\left(K+\frac{1}{4} w_{1}\right)$ theory has a set of flat directions on the Higgs branch where the matter field in the $(\square, \square)$ gets an expectation value, breaking the gauge group to $S p(R)_{D} \times S p(K-R) \times S p\left(K-R+\frac{1}{4} w_{1}\right)$, where $S p(R)_{D}$ is diagonally embedded in the two original gauge groups. This flat direction exists for any $R=1 \ldots K$ and corresponds to moving $R$ of the point-like instantons away from the $Z_{2}$ singularity. In particular, $S p(R)_{D}$ has exactly the standard matter content of $R$ small instantons away from the $Z_{2}$ orbifold singularity.

Despite this evidence for $\mathcal{M}_{H} \cong \mathcal{M}_{\text {Inst }}$ with the data (4.7), there is an important caveat which was already apparent in the work of [15]. In the analogous hyper-Kahler quotient construction of [18] for $U(N)$ instantons on ALE spaces, and in the example of the previous subsection, it was possible to blow up the orbifold singularity via Fayet-Iliopoulos terms in the hyper-Kahler quotient gauge theories. In the present situation, however, the $S p\left(v_{0}\right) \times S p\left(v_{1}\right)$ gauge theory does not have any overall $U(1)$ factor, as would be needed to add a Fayet-Iliopoulos term. Therefore, the hyper-Kahler quotient construction of $\mathcal{M}_{\text {Inst }}$ discussed here only applies when the $Z_{2}$ orbifold singularity is not blown up; the corresponding blowing up mode is missing. While we will not find this missing mode, it should not be forgotten. Below we will argue that, in the physical $S O(32)$ string, it nicely 
joins with 28 other "missing" moduli in a transition where 29 hypermultiplets are traded in for a tensor multiplet.

The $S p\left(v_{0}\right) \times S p\left(v_{1}\right)$ theory with the data (4.7) can not arise as a world volume gauge theory of D5 branes at an orbifold singularity. This is because, with the data (4.7), the anomaly (4.5) is non-zero. In fact, the coefficients of the deadly $\operatorname{tr} F_{\mu}^{4}$ terms are non-zero, so the theory has a sickness which can not possibly be cured, even by adding more fields. There is, though, a simple modification of the data (4.7) for which the $\operatorname{tr} F_{\mu}^{4}$ terms in (4.5) do drop out:

$$
v_{0}=K, \quad v_{1}=K+\frac{w_{1}}{4}-4 \quad \text { (Physical) }
$$

With this choice, the $\operatorname{tr} F_{0}^{4}$ term properly vanishes for $N=32$ and the coefficient of the $\operatorname{tr} F_{1}^{4}$ term is identically zero. Furthermore, the remaining terms in (4.5) are a perfect square and thus the anomaly can be cancelled by including a single tensor multiplet.

With the choice of data (4.9), the Higgs branch of the $S p\left(v_{0}\right) \times S p\left(v_{1}\right)$ gauge theory is no longer isomorphic to the moduli space of instantons on the $Z_{2}$ singularity; even their dimensions differ. Applying the general formulae (4.4) and (4.6), we see that Higgs branch of the theory with data (4.9) has 28 fewer hypermultiplet moduli than the moduli space of instantons. In addition, there is the missing blowing up mode mentioned above. All together, we are missing 29 hypermultiplets and need an extra tensor multiplet.

This trade between 29 hypermultiplets and a tensor multiplet is familiar in compactifications of string theory to six dimensions, where it is crucial that both make the same contribution to the purely gravitational anomaly [20,21]. The classic example is the small $E_{8}$ instanton theory: there is a "Higgs" branch, with 29 massless hypermultiplets, which touches a "Coulomb" branch, with a massless tensor multiplet, at a transition point in the moduli space [4]. This point is where the $E_{8}$ instanton is of zero size. Other examples of such transitions involving extra tensor multiplets have been found in a variety of string compactifications to $6 \mathrm{~d}$.

The picture that we have for what happens when $K$ D5 branes sit on the $Z_{2}$ orbifold singularity is the following: There is an interesting six dimensional quantum field theory living on the world volume. This theory has a moduli space with two branches: "Higgs" and "Coulomb," which join near the origin. The Higgs branch is isomorphic to the full moduli space of instantons on the orbifold, including a mode for blowing up the orbifold. Near the origin, with 28 of the instanton moduli and the blowing-up mode set to zero, the theory has a "Coulomb" branch with a massless tensor multiplet, whose real scalar component $\Phi$ 
can get an expectation value. On this Coulomb branch is the $S p(K) \times S p\left(K+\frac{1}{4} w_{1}-4\right)$ gauge theory with a tensor multiplet. This situation is quite analogous to that of the small $E_{8}$ instanton picture, with the $S p \times S p$ gauge theory super-imposed on the tensor multiplet of the Coulomb branch.

Consider for simplicity the case with $w_{1}=0$. In order for the above gauge theory to make sense, $K \geq 4$; for $K<4$ there is no Coulomb branch. For $K=4$ small instantons sitting on the $Z_{2}$ singularity, there is a Coulomb branch to a theory which is similar to the usual $S p(4)$, with matter $16 \cdot \square$, though without the usual $\square$ in the $\mathbf{2 7}+\mathbf{1}$ of $S p(4)$, and with an extra tensor multiplet. The missing $\mathbf{2 7}+\mathbf{1}$ means that all four instantons are locked at the $Z_{2}$ orbifold point. In addition, the mode for blowing up the $Z_{2}$ orbifold singularity is locked to zero. These modes get a mass along the Coulomb branch. In the place of these 29 missing hypermultiplets is the extra tensor multiplet. Exactly this situation was independently found recently by Aspinwall to occur in compactification of the heterotic string to six dimensions on $K 3$ [12], where it was found via $F$ theory.

Along the Coulomb branch, as in the discussion following (2.5), the cancellation of the remaining anomaly in (4.5) means that

$$
g_{0, e f f}^{-2}=g_{0, c l}^{-2}+\Phi, \quad g_{1, e f f}^{-2}=g_{1, c l}^{-2}-\Phi,
$$

where we absorbed a normalization constant into the normalization of $\Phi$. The theory has a single coupling constant parameter: $g_{0}^{-2}+g_{1}^{-2}$. This is sensible because this is precisely the coupling of the $S p(R)_{D}$ theory obtained upon moving $R$ of the point like instantons away from the singularity. We can set $g_{0, c l}^{-2}=0$ in (4.10), by choice of the origin of $\Phi$, and take the Coulomb branch to be $\langle\Phi\rangle \in R^{+}$. The $S p(K)$ theory can have a non-trivial RG fixed point at the origin of the Coulomb branch. On the other hand, the coupling $g_{1, \text { eff }}^{-2}$ of $S p\left(K+\frac{1}{4} w_{1}-4\right)$ has a "Landau pole" on the Coulomb branch at $\langle\Phi\rangle \geq g_{1, c l}^{-2}$. This reflects the fact that the $S p\left(K+\frac{1}{4} w_{1}-4\right)$ theory is free at long distances and more data, such as string theory, is needed in the ultraviolet to obtain a sensible theory. This aspect of the theory is inherited from the theory of small instantons away from singularities, which is also IR free.

The transition point between the Higgs and Coulomb branches is associated with "tensionless strings," which is interpreted as a signature of an interacting, local, RG fixed point. As discussed above, only the $S p(K)$ gauge group can have a non-trivial fixed point. At long distances, the $S p\left(K+\frac{1}{4} w_{1}-4\right)$ theory is IR free and thus becomes un-gauged. The theory at the fixed point is then $S p(K)$ with matter given by $(2 K+8) \cdot \square$, along with a tensor multiplet. The string theory construction proves the existence of RG fixed points at the origin of the Coulomb branch of these theories. 


\section{Small Instantons at $Z_{M}$ orbifold singularities with vector structure}

We now generalize the analysis of the previous section to arbitrary $Z_{M}$ orbifolds with vector structure. Let $w_{\mu}$ be the number of eigenvalues of $\rho_{\infty}$ equal to $e^{2 \pi i \mu / M}$ for $\mu=0 \ldots M-1$. Having $\rho_{\infty} \in S O(N)$ requires $w_{\mu}=w_{M-\mu}$ and $\sum_{\mu=0}^{M-1} w_{\mu}=N$. For $M$ even, having $\rho^{M}=1$ in $\operatorname{Spin}(N)$ actually requires $w_{\frac{M}{2}}$ to be a multiple of four.

The instanton number is given by

$$
I=K+\sum_{\mu=0}^{M-1} \frac{\mu(M-\mu)}{4 M} w_{\mu}
$$

where $K$ is an arbitrary integer and the last term is the contribution to the second Chern class of the non-trivial flat connection of $\rho_{\infty}$. The dimension of the moduli space of instantons with these physical data is given by an index theorem to be

$$
\operatorname{dim}\left(\mathcal{M}_{\text {Inst }}\right)=(N-2) I+\frac{1}{2}\left(\sum_{\mu, \nu=0}^{M-1} w_{\mu} w_{\nu} X_{\mu \nu}-\sum_{\mu=0}^{M-1} w_{\mu} X_{\mu, M-\mu}\right)
$$

where the last terms are the $\eta$ invariant, with $X_{\mu \nu}$ defined by

$$
X_{\mu \nu} \equiv \frac{1}{2 M} \sum_{k=1}^{M-1} \frac{e^{2 \pi i k(\mu-\nu) / M}-1}{2-2 \cos \left(\frac{2 \pi k}{M}\right)}=-\frac{|\mu-\nu|(M-|\mu-\nu|)}{4 M} .
$$

The relevant gauge theory depends on whether $M$ is even or odd; in either case we write $M=2 P$ or $M=2 P+1$. The two cases are given by, respectively, the "I.4" and "I.2" quiver diagrams described in sect. 4.4 of [15]. For $M=2 P$ the gauge theory is

$$
S p\left(v_{0}\right) \times U\left(v_{1}\right) \times U\left(v_{2}\right) \times \cdots \times U\left(v_{P-1}\right) \times S p\left(v_{P}\right), \quad M=2 P,
$$

with hypermultiplets $\frac{1}{2} w_{0} \cdot \square_{0}, \oplus_{j=1}^{P-1} w_{j} \cdot \square_{j}, \frac{1}{2} w_{P} \cdot \square_{P}$, and $\oplus_{j=1}^{P}\left(\square_{j-1}, \square_{j}\right)$ (subscripts label the gauge group). For $M=2 P+1$ the relevant gauge theory is

$$
S p\left(v_{0}\right) \times U\left(v_{1}\right) \times U\left(v_{2}\right) \times \cdots \times U\left(v_{P-1}\right) \times U\left(v_{P}\right), \quad M=2 P+1
$$

with hypermultiplets in the $\frac{1}{2} w_{0} \cdot \square_{0}, \oplus_{j=1}^{P} w_{j} \cdot \square_{j}, \oplus_{j=1}^{P}\left(\square_{j-1}, \square_{j}\right)$, and $\square_{P}$. It will be convenient in what follows to define $V_{\mu}$ for $\mu=0 \ldots M-1$ by $V_{\mu \leq P} \equiv \operatorname{dim}\left(\square_{\mu}\right)$ and $V_{\mu>P} \equiv V_{M-\mu}$; i.e. $V_{0} \equiv 2 v_{0}, V_{i<P} \equiv v_{i}$, and $V_{P} \equiv 2 v_{P}\left(V_{P} \equiv v_{P}\right)$ for $M=2 P$ $(M=2 P+1)$. 
The anomaly (2.1) for the above theories is given, using (2.3), by

$$
\mathcal{A}=\frac{1}{2} \sum_{\mu=0}^{M-1}\left(\widetilde{C}_{\mu \nu} V_{\nu}-w_{\mu}+D_{\mu}\right) \operatorname{tr} F_{\mu}^{4}+3 \sum_{j=1}^{P}\left(\operatorname{tr} F_{j-1}^{2}-\operatorname{tr} F_{j}^{2}\right)^{2}
$$

$F_{\mu}$ for $\mu>P$ is defined by $F_{\mu} \equiv F_{M-\mu}, \widetilde{C}_{\mu \nu} \equiv 2 \delta_{\mu \nu}-a_{\mu \nu}$ is the Cartan matrix of the extended $S U(M)$ Dynkin diagram, and $D_{\mu} \equiv 8\left(2 \delta_{\mu, 0}+\delta_{\mu, P}+\delta_{\mu, M-P}\right)$. We will also be interested in the Higgs branch; when there is sufficient matter to completely Higgs the gauge group, as will be the case below, its dimension can be written as

$$
\operatorname{dim}\left(\mathcal{M}_{H}\right)=\frac{1}{2} V_{\mu} w_{\mu}-\frac{1}{4} \widetilde{C}_{\mu \nu} V_{\mu} V_{\nu}-\frac{1}{2}\left(V_{0}+V_{P}\right) .
$$

Throughout, repeated greek indices are summed from 0 to $M-1$.

The above $V_{\mu}$ will be related to the $w_{\mu}$ and $K$ by $V_{0}=2 K$ and $\widetilde{C}_{\mu \nu} V_{\nu}=w_{\mu}-u_{\mu}$ for some $u_{\mu}$ satisfying $u_{\mu} n_{\mu}=w_{\mu} n_{\mu}=N$, with $n_{\mu} \equiv 1$ for all $\mu$. Thus,

$$
V_{0}=2 K, \quad V_{i \neq 0}=2 K+\sum_{j=1}^{M-1} C_{i j}^{-1}\left(w_{j}-u_{j}\right)
$$

with $C_{i j}^{-1}$ the inverse $S U(M)$ Cartan matrix, given by $C_{i<j}^{-1}=i(M-j) / M$. With (5.8),

$$
\frac{1}{M} \sum_{\mu=0}^{P} v_{\mu}=\frac{1}{2 M} v_{\mu} n_{\mu}=K+\sum_{\mu=0}^{M-1} \frac{\mu(M-\mu)}{4 M}\left(w_{\mu}-u_{\mu}\right)=I-\sum_{\mu=0}^{M-1} \frac{\mu(M-\mu)}{4 M} u_{\mu}
$$

Also, It follows from (5.8) and some straightforward but tedious manipulations that

$$
\operatorname{dim}\left(\mathcal{M}_{\text {Inst }}\right)=\operatorname{dim}\left(\mathcal{M}_{H}\right)-\sum_{j=1}^{M-1}\left(\left(u_{\nu} n_{\nu}-u_{0}\right) X_{0, j}+\frac{1}{2} C_{P, j}^{-1}\right) u_{j}+\frac{1}{2} \sum_{i, j=1}^{M-1} u_{i} u_{j} X_{i j} .
$$

We conjecture that $\mathcal{M}_{H} \cong \mathcal{M}_{\text {Inst }}$ for $u_{\mu}=N \delta_{\mu, 0}$ in (5.8). Explicitly,

$$
\left.V_{\mu \leq P}=2 K+\sum_{\nu=0}^{P} \min (\mu, \nu) W_{\nu} \quad \text { (Hyper }- \text { Kahler quotient }\right)
$$

where $W_{\mu<P} \equiv w_{\mu}$ and $W_{P} \equiv w_{P}\left(W_{P} \equiv \frac{1}{2} w_{P}\right)$ for $M$ odd (even). In this case, (5.9) is simply the instanton number, which is quite natural in terms of considering the instantons on the $M$-fold cover of the orbifold. In addition, in this case the gauge group (5.4) can be completely Higgsed and it follows from (5.10) that $\operatorname{dim}\left(\mathcal{M}_{H}\right)=\operatorname{dim}\left(\mathcal{M}_{\text {Inst }}\right)$. 
Note that the gauge groups (5.4) and (5.5) have $M-P-1$ overall $U(1)$ factors to which Fayet-Iliopoulos terms can be coupled. Adding such terms corresponds to turning on blowing-up moduli of the orbifold. However, because the $Z_{M}$ orbifold should have $M-1$ blowing up moduli, we see that $P$ of the blowing up modes are locked at zero. As in the previous section, these $P$ missing modes will be remembered below.

The theory with data (5.8) for $u_{\mu}=N \delta_{\mu 0}$, however, can not arise in the world volume of D5 branes on the orbifold singularity. This is because this theory has a deadly $\operatorname{tr} F_{P}^{4}$ anomaly term with non-zero coefficient. There is an obvious modification of (5.8), though, for which all $\operatorname{tr} F_{\mu}^{4}$ terms do (5.6) vanish: $u_{\mu}=D_{\mu} \equiv 8\left(2 \delta_{\mu 0}+\delta_{\mu P}+\delta_{\mu, M-P}\right)$; which requires $N=32$, as expected. More explicitly, with this modification (5.11) becomes

$$
V_{\mu \leq P}=2 K+\sum_{\nu=0}^{P} \min (\mu, \nu) W_{\nu}-8 \mu \quad(\text { Physical })
$$

With this choice of data, (5.6) becomes

$$
\mathcal{A}=3 \sum_{j=1}^{P}\left(\operatorname{tr} F_{j-1}^{2}-\operatorname{tr} F_{j}^{2}\right)^{2},
$$

which can be nicely cancelled by coupling the theory to $P$ tensor multiplets.

With the choice of data (5.12), (5.9) gives

$$
\frac{1}{M} \sum_{\mu=0}^{P} v_{\mu}=I-\left(M-\frac{M-2 P}{M}\right) .
$$

This difference has a natural interpretation: the $v_{\mu}$ make up the difference between the instanton number and that of the standard embedding. With the standard embedding, one should have $I_{\text {s.e. }}=\chi$, the Euler character. Actually, there are two possible choices for $\chi$ associated with the $Z_{M}$ orbifold: that of the bulk, which is $\chi_{b u l k}=M-M^{-1}$, and one which includes a contribution associated with the boundary at infinity, $\chi_{\text {total }}=$ $\chi_{\text {bulk }}+\chi_{\text {boundary }}=M$. For $M=2 P+1$, the $v_{i}$ make up the difference between the instanton number $I$ and $I_{\text {s.e. }}=\chi_{\text {bulk }}$. For $M=2 P$ the $v_{i}$ make up the difference between $I$ and $I_{\text {s.e. }}=\chi_{\text {total }}$. The fact that all $v_{\mu}=0$ when $I=\chi_{b u l k}$ for $M=2 P+1$ is related to the result that there are no five-branes in compactification of type I on a $Z_{3}$ orientifold $K 3$ [22]. 
In addition, with (5.12) it is no longer true that $\mathcal{M}_{H} \cong \mathcal{M}_{\text {Inst }}$. Indeed, even their dimensions differ: as seen from (5.10),

$$
\operatorname{dim}\left(\mathcal{M}_{H}\right)=\operatorname{dim}\left(\mathcal{M}_{\text {Inst }}\right)-28 P
$$

we are missing $28 P$ hypermultiplet moduli. Combining these with the $P$ missing blowingup modes mentioned above, we see that a transition has occurred where $29 P$ hypermultiplets have been traded for $P$ tensor multiplets. This is perfect, because $P$ tensor multiplets is precisely what we needed above to cancel the anomaly (5.13).

We thus conjecture that the theories (5.4) or (5.5) with the data (5.12), and $P$ tensor multiplets, arise as a "Coulomb branch" of small instantons on orbifold singularities with vector structure. Note that the Coulomb branch can only exist when all (5.12) satisfy $V_{\mu} \geq 0$. In particular, for $\rho_{\infty}=1$ (i.e. $w_{\mu}=32 \delta_{\mu, 0}$ ), the condition for there to be a Coulomb branch is $K \geq 4 P$.

Cancelling (5.13) via (2.5), the effective gauge couplings on the Coulomb branch satisfy

$$
g_{i, e f f}^{-2}=g_{i, c l}^{-2}+\Phi_{i+1}-\Phi_{i}, \quad i=0 \ldots P
$$

where $\Phi_{0} \equiv \Phi_{P+1} \equiv 0$ and normalization constants are absorbed into the normalization of $\Phi_{i}$. There is thus a single parameter coupling, $\sum_{i=0}^{P} g_{i}^{-2}$; this is the coupling of the $S p(R)$ theory, obtained along a flat direction, which is associated with moving $R$ instantons away from the singularity. By a choice of the origin of the $\Phi_{i}$, it is possible to take $g_{i<P, c l}^{-2}=0$ in (5.16). The Coulomb branch is then given by the wedge in $R^{P}, 0 \leq \Phi_{1} \leq \Phi_{2} \ldots \leq \Phi_{P}$, with $g_{i<P, e f f}^{-2} \geq 0$ in the entire wedge. On the other hand, $g_{P, e f f}^{-2}$ hits a Landau pole when $\left\langle\Phi_{P}\right\rangle \geq g_{P, c l}^{-2}$. This is a reflection of the fact that the last $S p\left(v_{P}\right)$ factor in (5.4) or $U\left(v_{P}\right)$ factor in (5.5) is IR free and UV incomplete. This property is inherited from the theory discussed in sect. 3 .

At the origin of the Coulomb branch, $\left\langle\Phi_{i}\right\rangle=0$, there is an interacting RG fixed point associated with (5.4) or (5.5) and the data (5.12). However, because the last $S p\left(v_{P}\right)$ or $U\left(v_{P}\right)$ factor in (5.4) or (5.5) is IR free, this factor is not gauged at the fixed point obtained at long distances. Summarizing, then, the fixed point theories have gauge group $S p\left(v_{0}\right) \times \prod_{i=1}^{P-1} U\left(v_{i}\right)$, with data (5.12), hypermultiplets $\frac{1}{2} w_{0} \cdot \square_{0}, \oplus_{j=1}^{P-1}\left(w_{j}+V_{P} \delta_{j, P-1}\right) \cdot \square_{j}$, $\oplus_{j=1}^{P-1}\left(\square_{j-1}, \square_{j}\right)$, and $P$ tensor multiplets. The string theory construction in terms of branes at orbifold singularities shows that these fixed points really exist. 


\section{Small Instantons at $Z_{2 P}$ orbifold singularities without vector structure}

We now briefly turn to the cases without vector structure. For $M=2 P, \rho_{\infty}$ has $w_{i}$ eigenvalues $e^{i \pi(2 i-1) / 2 P}, i=1 \ldots 2 P$, with $w_{2 P+1-i}=w_{i}$ and $\sum_{i=1}^{2 P} w_{i}=2 \sum_{i=1}^{P}=N$ for $\rho_{\infty} \in S O(N)$. It is expected that the relevant gauge theory is that of the "type I5" quiver diagrams of [15]. The gauge group is $\prod_{i=1}^{P} U\left(v_{i}\right)$ and the matter content is $\oplus_{i=1}^{P} w_{i} \cdot \square_{i}, \oplus_{i=1}^{P-1}\left(\square_{i}, \square_{i+1}\right), \square_{1}$, and $\square_{P}$. Note that, as there are only $P U(1)$ factors to which Fayet-Iliopoulos terms can be coupled, there are $P-1$ missing blowing up modes.

The anomaly (2.1) is found to be

$$
\mathcal{A}=\frac{1}{2} \sum_{i=1}^{2 P}\left(\sum_{j=1}^{2 P} \widetilde{C}_{i j} v_{j}-w_{i}+D_{i}\right) \operatorname{tr} F_{i}^{4}+3 \sum_{r=1}^{P-1}\left(\operatorname{tr} F_{r}^{2}-\operatorname{tr} F_{r+1}^{2}\right)^{2}
$$

where $\widetilde{C}_{i j}$ is the Cartan matrix for the extended $S U(2 P)$ Dynkin diagram, $F_{i>P} \equiv$ $F_{2 P+1-i}, v_{i>P} \equiv w_{2 P+1-i}$, and $D_{i} \equiv 8\left(\delta_{i, 1}+\delta_{i, P}+\delta_{i, 2 P}+\delta_{i, P+1}\right)$. These theories thus have an anomaly which can be cancelled with $P-1$ tensor multiplets provided

$$
\sum_{j=1}^{2 P} \widetilde{C}_{i j} v_{j}=w_{i}-D_{i}
$$

Note that this properly gives $N=32$.

Cancelling (6.1) via (2.5), the effective gauge couplings are,

$$
g_{i, e f f}^{-2}=g_{i, c l}^{-2}+\Phi_{i}-\Phi_{i-1}, \quad i=1 \ldots P
$$

with $\Phi_{0} \equiv \Phi_{P} \equiv 0$ and normalization factors absorbed into the normalization of the $\Phi_{i}$. There is thus a single coupling parameter, $\sum_{i=1}^{P} g_{i}^{-2}$, which is that of the $S p(R)$ gauge theory of small instantons away from the singularity. By choice of the origin of the $\Phi_{i}$, we can take $g_{i<P, c l}=0$ in (6.3) and the Coulomb branch to be given by the wedge in $\mathbf{R}^{P-1}$ : $0 \leq \Phi_{1} \leq \Phi_{2} \cdots \leq \Phi_{P-1}$. The $g_{i<P, e f f}^{-2} \geq 0$ everywhere on the Coulomb wedge. On the other hand, $g_{P, \text { eff }}^{-2}$ hits a Landau pole at $\left\langle\Phi_{P}\right\rangle \geq g_{P, c l}^{-2}$. This corresponds to the fact that the last $U\left(v_{P}\right)$ factor is IR free.

We conjecture that these theories arise as the Coulomb branch of the world volume theory of D5 branes on orbifold singularities without vector structure. Some examples were explicitly constructed via orientifold techniques in [22,23]: the " $Z_{4}^{A}$ " orientifold there gave the $P=2$ case, with $v_{i}=w_{i}=8$, and the " $Z_{6}^{A}$ " orientifold gave the $P=3$ case, with $v_{i}=w_{i}, w_{1}=w_{3}=4, w_{2}=8$. These restrictions came from compactifying on a 
compact $K 3$. We conjecture that all 6 d gauge theories of the above type satisfying (6.2) can be obtained in the world volume of D5 branes at (non-compact) orbifold singularities without vector structure.

The $\prod_{i=1}^{P-1} U\left(v_{i}\right)$ part of the above theories have non-trivial RG fixed points at the origin of the Coulomb branch. The $U\left(v_{P}\right)$ part is IR free and thus un-gauged at long distances]. The string theory construction shows that all of these fixed points really exist.

\section{Acknowledgments}

I would like to thank J. Blum, M. Douglas, N. Seiberg, and E. Witten for useful discussions. The work of K.I. is supported by NSF PHY-9513835, the W.M. Keck Foundation, an Alfred Sloan Foundation Fellowship, and the generosity of Martin and Helen Chooljian.

5 In all of the examples, by changing the sign of the range of the $\left\langle\Phi_{i}\right\rangle$ on the Coulomb branch, the first rather than the last gauge group can be taken to be the one which is IR free. For the gauge theories (5.4) and those discussed in this section, this operation simply leads to the same fixed point theories. On the other hand, the fixed point theories described in this section could, alternatively, have been obtained from (5.5) via this operation. 


\section{References}

[1] J. Polchinksi, "TASI Lectures on D-Branes," hep-th/9611050.

[2] E. Witten, "Small Instantons in String Theory," hep-th/9511030, Nucl. Phys. B 460 (1995) 541.

[3] O. Ganor and A. Hanany, "Small E(8) Instantons and Tensionless Noncritical Strings," hep-th/9602120.

[4] N. Seiberg and E. Witten, "Comments on String Dynamics in Six Dimensions," hepth/9603003, Nucl. Phys. B 471 (1996) 121.

[5] M.J. Duff, H. Lu, and C.N. Pope, "Heterotic Phase Transitions and Singularities of the Gauge Dyonic String," hep-th/9603037, Phys. Lett. B 378 (1996) 101.

[6] E. Witten, "Physical Interpretation of Certain Strong Coupling Singularities," hepth/9609159, Mod. Phys. Lett. A11 (1996) 2649.

[7] E. Witten, "Some Comments on String Dynamics," hep-th/9507121, Proc. of Strings '95, editors I. Bars et. al., World Scientific, 1996.

[8] K. Intriligator and N. Seiberg, "Mirror Symmetry in Three Dimensional Gauge Theories," hep-th/9607207, Phys. Lett. B 387 (1996) 513.

[9] N. Seiberg, "Five Dimensional SUSY Field Theories, Non-trivial Fixed Points, and String Dynamics," hep-th/9608111.

[10] O. J. Ganor, D. R. Morrison, and N. Seiberg, "Branes, Calabi-Yau Spaces, and Toroidal Compactification of the $N=1$ Six Dimensional $E_{8}$ Theory," hep-th/9610251.

[11] N. Seiberg, "Non-trivial Fixed Points of The Renormalization Group in Six Dimensions," hep-th/9609161.

[12] P. S. Aspinwall, "Point-like Instantons and the $\operatorname{Spin}(32) / Z_{2}$ Heterotic String," hepth/9612108.

[13] M. Berkooz, R.G. Leigh, J. Polchinski, J. Schwarz, N. Seiberg, and E. Witten, "Anomalies, Dualities, and Topology of $D=6 N=1$ Superstring Vacua," hep-th/9605184, Nucl. Phys. B 475 (1996) 115.

[14] E. G. Gimon and Polchinski, "Consistency Conditions for Orientifolds and DManifolds," hep-th/9601038, Phys. Rev. D 54 (1996) 1667.

[15] M. Douglas and G. Moore, "D-Branes, Quivers, and ALE Instantons," hep-th/9603167.

[16] J. H. Schwarz, "Anomaly-Free Supersymmetric Models in Six Dimensions," hepth/9512053, Phys. Lett. B 371 (1996) 223.

[17] A. Sagnotti, "A Note on the Green-Schwarz Mechanism in Open String Theory," hep-th/9210127, Phys. Lett. B 294 (1992) 196.

[18] P. B. Kronheimer and H. Nakajima, "Yang-Mills Instantons on ALE Gravitational Instantons," Math. Ann. 288 (1990) 263.

[19] P.B. Kronheimer, Jour. Differential Geometry, 29 (1989) 665. 
[20] L. Alvarez-Gaume' and E. Witten, "Gravitational Anomalies," Nucl. Phys. B 234 (1983) 269.

[21] M. Duff, R. Minasian, and E. Witten, "Evidence for Heterotic/Heterotic Duality," hep-th/9601036, Nucl. Phys. B 465 (1996) 413.

[22] E. G. Gimon and C. V. Johnson, "K3 Orientifolds," hep-th/9604129, Nucl. Phys. B 477 (1996) 715.

[23] A. Dabholkar and J. Park, "Strings on Orientifolds," hep-th/9604178, Nucl. Phys. B 477 (1996) 701. 